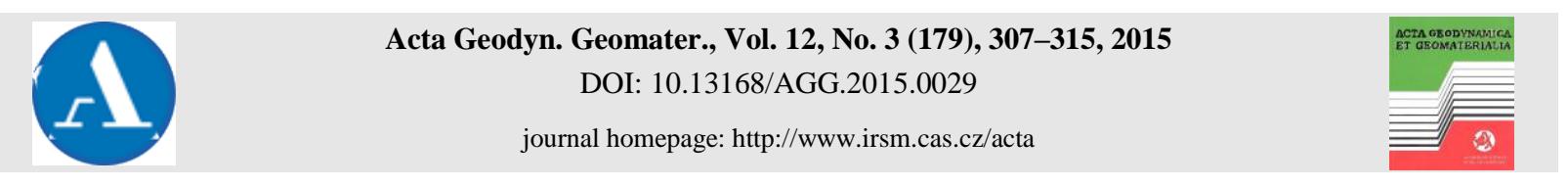

ORIGINAL PAPER

\title{
COMPARISON OF SELECTED DATA ACQUISITION METHODS FOR GNSS TERRAIN OBSTACLES MODELING
}

\author{
Renata PELC-MIECZKOWSKA ${ }^{1)}$, Joanna JANICKA ${ }^{2)}$, \\ Michal BEDNARCZYK ${ }^{1)}$ and Dariusz TOMASZEWSKI ${ }^{2)}$
}

${ }^{1)}$ Department of Land Surveying and Geomatics, University of Warmia and Mazury in Olsztyn, Heweliusza 12, 10-727 Olsztyn, Poland

${ }^{2)}$ Institute of Geodesy, University of Warmia and Mazury in Olsztyn, Oczapowskiego 1, 10-719 Olsztyn, Poland

*Corresponding author's e-mail: renata.pelc@uwm.edu.pl

\begin{tabular}{|c|c|}
\hline ARTICLE INFO & ABSTRACT \\
\hline Article history: & \multirow{6}{*}{$\begin{array}{l}\text { For satellite measurements, both in navigation and surveying, unobstructed visibility of at least } \\
\text { three ( } 2 \mathrm{D} \text { positioning), four ( } 3 \mathrm{D} \text { positioning) or five (RTK) satellites is essential. In urban areas } \\
\text { or in the vicinity of trees knowledge of shape and location of the terrain obstacles may determine } \\
\text { the success of GNSS positioning. The aim of the presented research is to investigate the } \\
\text { suitability of the use of laser scanning and digital hemispherical photography for modeling } \\
\text { terrain obstacles over the GNSS measurement points. The accuracy of data acquisition methods } \\
\text { was examined by taking the coordinates from tacheometric measurement as the reference. }\end{array}$} \\
\hline Received 20 January 2015 & \\
\hline Accepted 17 June 2015 & \\
\hline Available online 17 July & \\
\hline Keywords: & \\
\hline GNSS & \\
\hline
\end{tabular}

Obstacles modeling

Hemispherical photography

Fish-eye lens

Terrestrial laser scanning

\section{INTRODUCTION}

Nowadays, thanks to the large number of satellites in GNSS systems constellations and the rapid development of Global Navigation Satellite Systems Permanent Station Networks, the satellite measurement techniques are widely used not only in geodetic surveying but also in environmental monitoring or navigation. Moreover, satellite positioning is often applied in more complex, multifunctional systems, such as, urban traffic monitoring system for taxi corporation, where many other technologies (eg. GIS mobile indicators, Internet communication), were also used (Janowski et at., 2014). Such measurements became simple, quick and cheap, that is why the range of their application is extending to areas that were not available for them so far, such as mountain valleys, urban canyons or wooded areas. In such severe environments some obstructions like huge terrain denivelations, high buildings, bridges or trees branches and canopies, prevent GNSS satellite signal reception. Such blockage affects proper ambiguity of phase measurements solving and significantly decreases of probability and reliability of coordinates determination (Bakuła et. al., 2012; Bakuła et. al., 2011; Bakuła et al., 2009). Visibility of required number of satellites is constraint for successful GNSS positioning. Therefore it is important to know in advance where and when signal will be concealed by obstacles and when the signals from a sufficient number of satellites will be directly available. Hence, in case of difficult measurement conditions, it is essential to take into account the shape and type of terrain obstacles during the mission planning process.
The process of mission planning essentially consists of two tasks: determination of predicted position of particular satellites referred to GNSS receiver (Parkinson, 1996), which is obvious and generation obstruction diagram or model. For over 20 years, several methods of the position and shape of the curtain determining have been proposed. The earliest of them assumed the use of engineer's theodolite (Krohn et al., 1988) or so called floating lines method (Boulianne et al., 1996) for direct field measurement. Tacheometry is definitely a very accurate method but the main disadvantage of this methods is that it is extremely time-consuming and, what is more, there is some technical inconvenience if high elevations should be measured. Most recently developed methods are based on Digital Terrain Model (DTM) or Digital Surface Model (DSM) derived from various sources and GIS procedures (Federici et al., 2013; D'Inca et al., 2004; Taylor et al., 2007; Groves, 2011; Ackermann et al., 2013). The main benefit of applying DTM or DSM in mission planning process is that such model covers not just single measurement point surrounding but also some area around measurement point which makes it suitable for kinematic survey. On the other hand, using these methods requires $a$ priori determination of measurement point coordinates with relatively high accuracy. In this paper two further methods of direct data acquisition for terrain obstacles modeling are introduced and compared. First of them is terrestrial laser scanning and the second one is digital, hemispherical photography. Both of them provide the obstruction diagram in situ and are also expected to be faster and cheaper than tacheometry. Terrestrial laser scanning 
allows for fast acquisition of 3D information needed to represent objects that are complex in shape and whose measurement with use of conventional methods would be very difficult or impossible. Hemispherical image offers the advantage of providing spatially resolved information about measurement point neighborhood and that is why it could be a powerful tool for GNSS obstruction diagram generation (Colcord, 1989; Pelc-Mieczkowska, 2014).

Laser scanning is an active data acquisition system that allows for the precise determination of the shape and geometric relationships between objects in the scanner range. Terrestrial laser scanner measures the distance, horizontal and vertical angle between the device and the test object (Fig. 1).

Then, on the basis of these data, coordinate $X, Y$, $Z$ of each measured point are calculated, creating so called point cloud.

The basic elements of the scanner are the rotating mirrors. Mirrors rotate in the vertical plane. At the same time the scanner rotates around its own

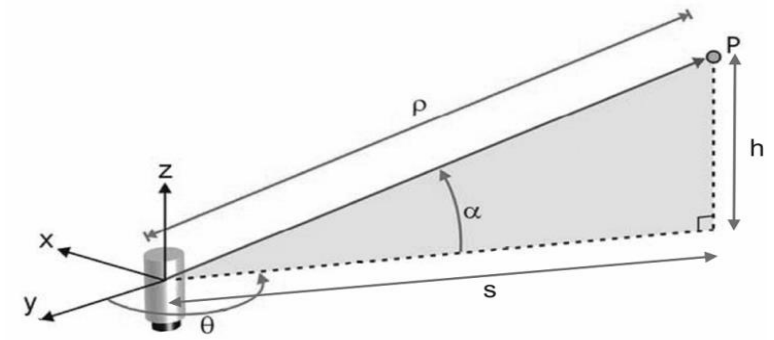

Fig. 1 The principle of laser scanner measurement.

axis. Horizontal rotation around the axis of the scanner allow to visualize the space in the range of $360^{\circ}$ but the vertical field of view is limited to $270^{\circ}$. However, the main advantages of terrestrial laser scanning are

- high speed data acquisition in a short time (several tens of thousands of points per second),

- high millimeter accuracy (range, 1-10 mm),

- resistance to errors caused by the observer,

- measurements in a difficult or impossible conditions for conventional measurement techniques.

On the other hand there are a lot of restrictions of this method like limited measuring range or the need to deal with large data sets.

Hemispherical photography, carried out with fish-eye lenses for instance, allowing for imaging a large area of surrounding space in just single picture. Because of extremely wide field of view, fish-eye lenses are widely and successfully used in different domains such as forestry (Beckschaefer et al., 1996), the study of plant canopies (Gonsamo et al., 2013), meteorology (Bulliner et al., 2013), mapping confined spaces (Boulianne et al., 1997) and site satellite window diagram direct determining. Vertically oriented and centered over GNSS measurement point, fish-eye photography produces terrain obstacles model in which points are described by azimuth and elevation pair of angles.

Using fish-eye lenses for any measurement purpose, a large amount of distortion should be taken into consideration, modeled and mitigated. A fair approximation for most conventional narrow-angle and even wide-angle lenses is the pinhole camera model which fulfilling the perspective projection described by following formula: $r=f \tan \Theta$, where $\Theta$ refers to the angle between the optical axis and the incoming ray, $r$ is the distance between the imagine point and the principal point and $f$ is a focal length. Because, in perspective projection it is impossible to project entire hemisphere on a fine image plane, fisheye lens model fulfills the principle that, in the ideal case, the distance between image point and principal point is linearly dependent on the angle of incidence of incoming ray (Fig. 2) (Schwalbe, 2005). The images obtained with the use of physical lenses are affected by a number of effects that depart from a linear perspective projection. Those include the basic lens model, radial distortion, tangential distortion and movement of the entrance pupil (Gennery, 2006).

Fish-eye cameras are designed to map the whole hemispherical field in front of the camera thus fisheye lenses mostly obey one of the following projection models (Fig. 3):

a) $r=2 f \tan \frac{\Theta}{2}$ - stereographic projection,

b) $r=f \Theta$ - equidistance projection,

c) $r=2 f \sin \frac{\Theta}{2}$ - equisolid angle projection,

d) $r=2 f \sin \Theta$ - orthogonal projection (Kannala et. al., 2006).

Kannala suggests that the most common model is the equidistance projection.

Previously mentioned camera models usually assume a fixed position of the entrance pupil. In the

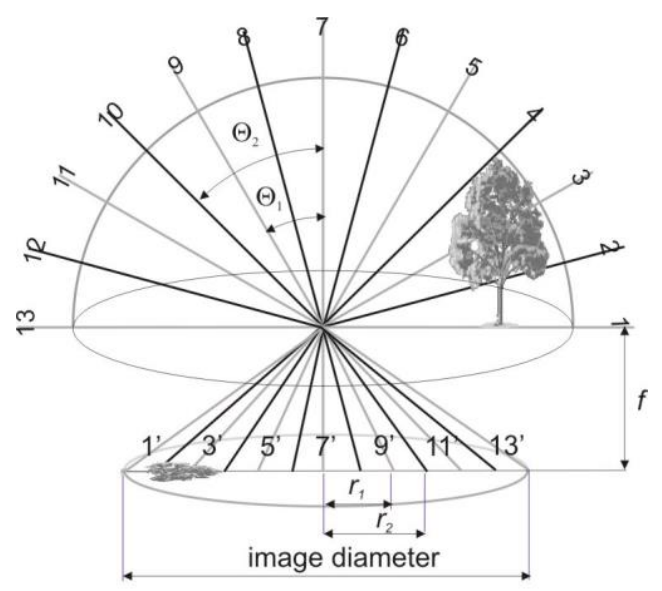

Fig. 2 The principal of fish-eye camera model. 
case of wide-angle lenses the entrance pupil point varies with the off-axis angle of the incident ray. The empirically determined amount of this movement assumes values within $2 \mathrm{~cm}$. The knowledge about the position of the entrance pupil point is particularly important when stitching panoramic images from series of individual photos. In case of elevation and azimuth measurement based on single picture, the movement of the entrance pupil point is relevant only if the line of site is short. For distances above 2 meters the influence of the entrance pupil point movement is less than 0.07 grad $^{1}$ and can be neglected (Fig. 4).

The real lenses do not exactly follow the designed projection model. For the determination of
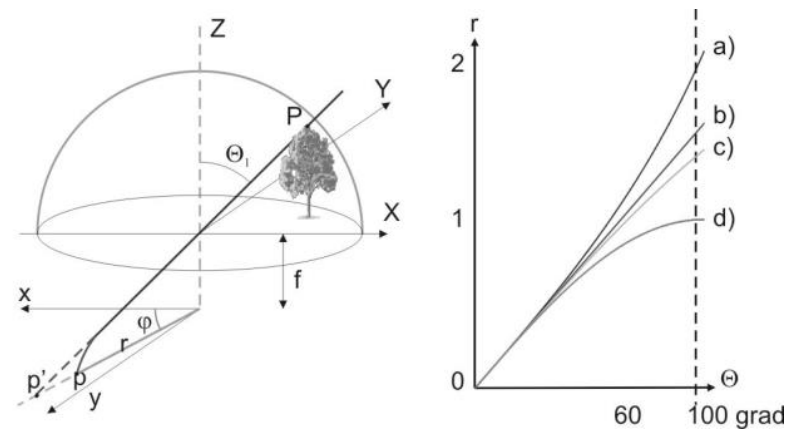

Fig. 3 Fish eye camera model and projections (based on Kannala, 2006).

correct azimuth and elevation angles at hemispherical pictures, not only the knowledge of proper projection is important but also the radial and tangential distortion, which are radially symmetrical, also should be considered. The value of distortion at a given image point is a function of incoming ray angle of incidence. The most popular radial distortion model is the odd-order polynomial model (Gennery, 2006; Kannala, 2006) in general form:

$$
r(\Theta)=k_{1} \Theta+k_{2} \Theta^{2}+k_{3} \Theta^{5}+k_{4} \Theta^{7}+k_{5} \Theta^{9}+\cdots
$$

Kannala indicates that first five terms, up to the ninth power of $\Theta$, give enough degree of freedom for good approximation of different projection curver. Thus, the radially symmetric part of our model contains five polynomial coefficients, $k_{1}, k_{2}, \ldots, k_{5}$, which should be determined in the calibration process.

Tangential distortion is assigned to inclination and decentering of internal lens elements. Under its influence, every object in the picture is moved from theoretical position in a direction perpendicular to radial distortion direction. In good quality lenses the value of tangential distortion is about 10 times less than radial distortion (Luhmann et al., 2006; Remondino et al., 2006), so its effects are mostly negligible (Hugemann, 2010). Thus in this work tangential distortion wasn't taken into consideration.

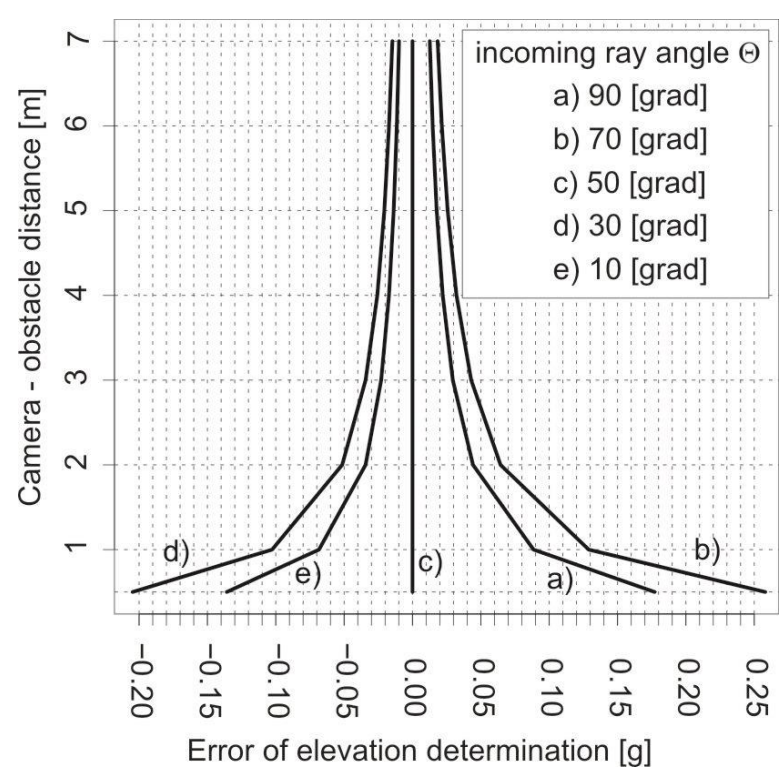

Fig. 4 The entrance pupil point movement influence on error of elevation determination.

The aim of the presented research is to investigate the suitability of the use of laser scanning and digital hemispherical photography for modeling of terrain obstacles over the GNSS measurement points. That task involves calibration of camera-lens set, the preparation of the test object, tachymetric measurement and finally laser scanning measurement and hemispherical photography. The accuracy of data acquisition methods was examined by taking the coordinates from tachymetric measurement as the reference.

\section{METHODOLOGY}

For the purpose of comparison of data acquisition methods, dedicated test object was prepared. This object consists of single fixed measurement point and 41 control points marked by targets. The control points are placed as they form four zenith-horizon lines at horizontal angle 0, 100, 200 and 300 grads respectively. The differences in elevation between neighboring control points on the respective line are equal and are 10 grads. The control points were set out by tacheometer Leica TS15 equipped with laser pointer. In the next step, elevation el and horizontal angle $\mathrm{Hz}$ of each target was measured by tacheometer Leica TS15 with integrated camera. These coordinates were regarded as reference for further tests.

First over the point where the tacheometer measurements were made, the Leica C10 laser scanner was placed. The center of the scanners telescope was set at the same height of as in the case of tacheometers measurements. Afterwards all previously delineated stamps were scanned as it can be seen in Figure 5. 
On the basis of made measurement, the coordinates of all scanned points were obtained. The coordinates were in the local frame defined by the axes of the scanner. The next step was to determine which of the scanned points are at the marked stamps centers. This task was carried out with the use of the laser beam intensity parameter. This parameter describes the amount of light which has returned to the scanner after the distance measurement was made. Its value depends directly on the type of the material from which the beam was reflected (color, facture, fabric) and the angle of incidence. Marked stamps were red which allowed them to be clearly identified in the point cloud from the scanner. Subsequently the points nearest the circles centers were determined. On the basis of obtained coordinates the values of horizontal angle and elevation of all marked stamps were calculated. The calculated values were compared with the theoretical values established during tacheometry.

The fish-eye lens camera systems which have been used was Nikon D90 12.3 megapixel digital semi-professional camera with Sigma $8 \mathrm{~mm}$ f/3.5 EX DG circular fish-eye lens (180 degree field of view). The camera set was mounted on geodetic tripod and carefully centered over measurement point. The lens was oriented vertically and leveled. Using such prepared test set, four, 33 grads rotated, hemispherical pictures were made (Fig. 5).

At each picture two quantities were measured for each point. The first one is the radial distance $r$ between the image geometric center and the respective point, while the second one is a horizontal angle $\mathrm{Hz}$ construed as the angle between arbitrary fixed direction (in this experiment a line connecting the zenith point No.11 and the point No.1) and radius vector of the respective point. Then the elevations $\mathrm{el}$ of each of the survey marks were determined assuming equisolid angle projection model:

$$
e l=2 \arcsin \left(\frac{r}{2 f}\right),
$$

where $f$ is focal length. Thus position of each control point obtained from the image was determined by a pair of coordinates $(e l, H z)$.

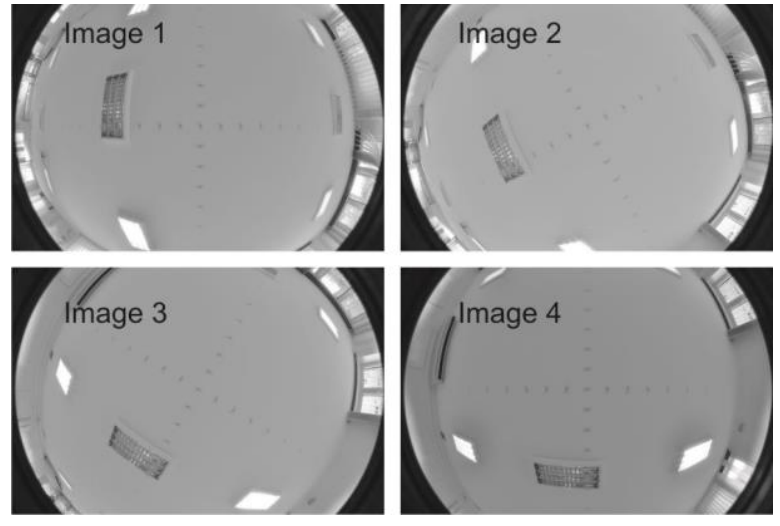

Fig. 6 Hemispherical pictures of test object.

As it was mentioned in introduction, not only proper projection model but also some model of radial and tangential distortion should be taken into consideration. In the presented research, distortions parameters are estimated based on least squares polynomial fitting. To determine polynomial coefficients empirically the calibration box with set of check lines was established (Fig. 6). The calibration box was designed in a way to ensure image points of intersection the vertical with horizontal lines along the entire radius of the lens. The distance between vertical lines equals $5 \mathrm{~mm}$ and line of site equals $156 \mathrm{~mm}$.

Because of the short lines of sight $(156 \mathrm{~mm}$ at zenith and $382 \mathrm{~mm}$ at horizon), the entrance pupil point position and its movement had to be taken into account. These quantities for Sigma $8 \mathrm{~mm}$ f/3.5 EX DG circular fish-eye lens were experimentally determined with low power laser beam technique. The value of this offset was referred to the position of golden ring marked on the lens and ranged from 0.5 $\mathrm{cm}$ to $2 \mathrm{~cm}$ (Fig. 7)

Based on determined entrance pupil point position, known line of site length and line-to-line distances, reference values of elevation of intersection points was calculated. The radial distance $r$ of those points images were also measured in the picture. Afterwards, elevations (el) of those points were calculated. Subsequently five polynomial coefficients: $k_{1}, k_{2}, k_{3}, k_{4}, k_{5}$, (equation (1)) were determined. The
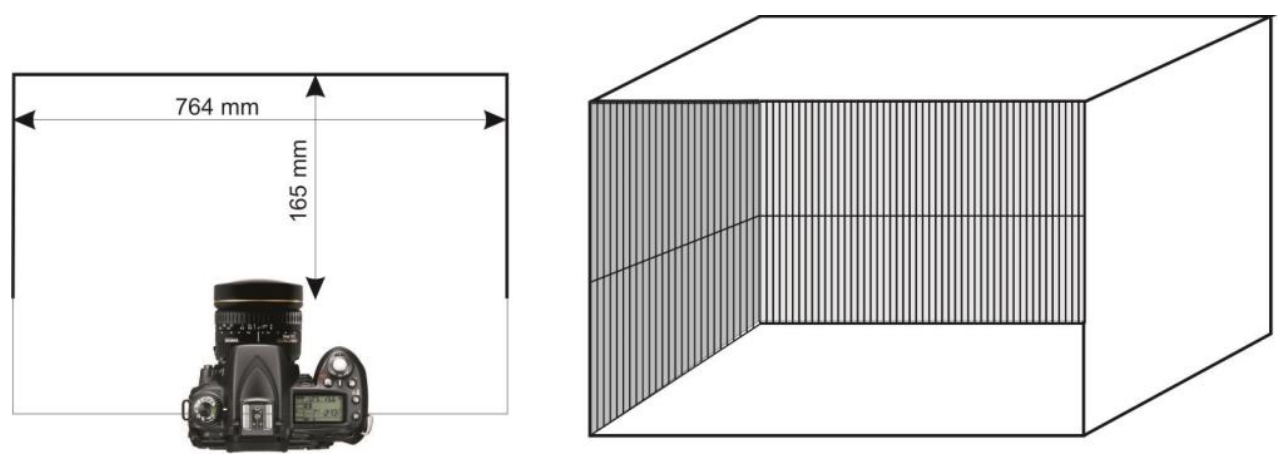

Fig. 7 Diagram of calibration box. 


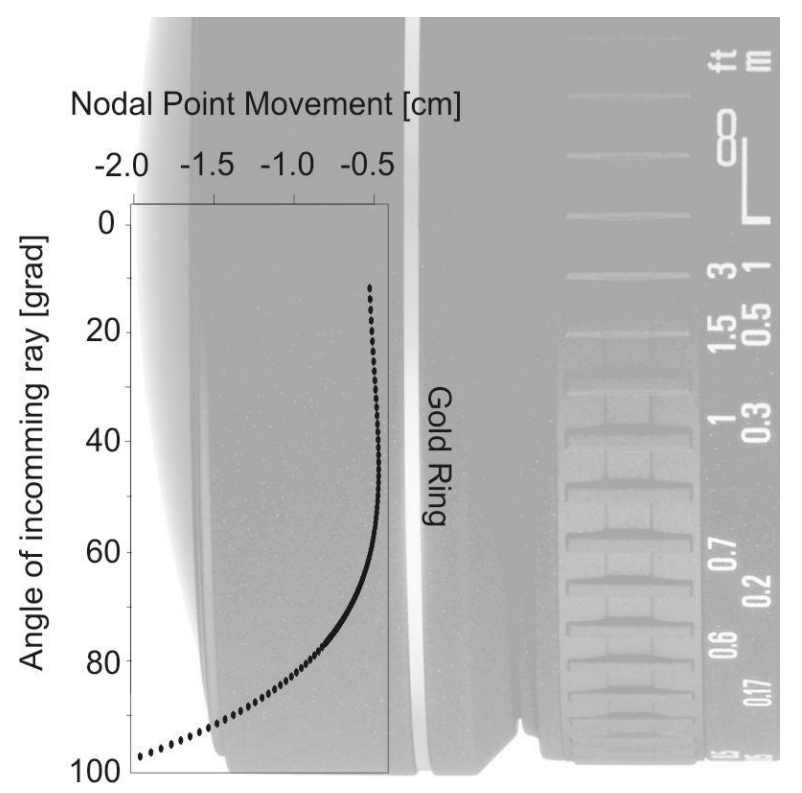

Fig. 8 The entrance pupil point movement for Sigma $8 \mathrm{~mm} \mathrm{f} / 3.5 \mathrm{EX}$ DG circular fish-eye lens.

coefficients of polynomial were calculated with 211 degrees of freedom on which the residual standard error was only 0.162 . Graph that shows the fitting of a polynomial is presented below (Fig. 9).

Calculated polynomial coefficients were applied to reduce the elevations $(e l)$ of the survey marks of test object.

\section{RESULTS}

As a result of conducted experiments three sets of $e l, \mathrm{~Hz}$ coordinates of survey marks were obtained. Coordinates obtained from tacheometry were used as a reference.

The first analyses were performed on the results of the laser scanner measurement. As it was mentioned previously, values of horizontal angle and elevation obtained from the scanner were differenced with reference values. The results performed analysis are presented below (Fig. 10)

As it can be seen in Table 1 horizontal angle residuals do not exceed 0.5667 grade and are in the range from -0.3461 grade to 0.5667 , but with standard deviation equal 0.1988. At the same time the maximum value of elevation residual is twice less than in the case of $\mathrm{Hz}$ angle and it is only 0.2517 . The extent to with the values of elevation angle residuals rade is also twice less and it is from -0.1336 to 0.2517 . Summarizing the obtained results it can be stated that laser scanning is a very accurate tool for terrain obstacles data acquisition.

Coordinates obtained from hemispherical photography (unreduced and reduced) were compared with reference coordinates, thus elevations and horizontal angles errors were calculated. Elevation angle errors values for unreduced readings were increasing from the center toward the edges of the

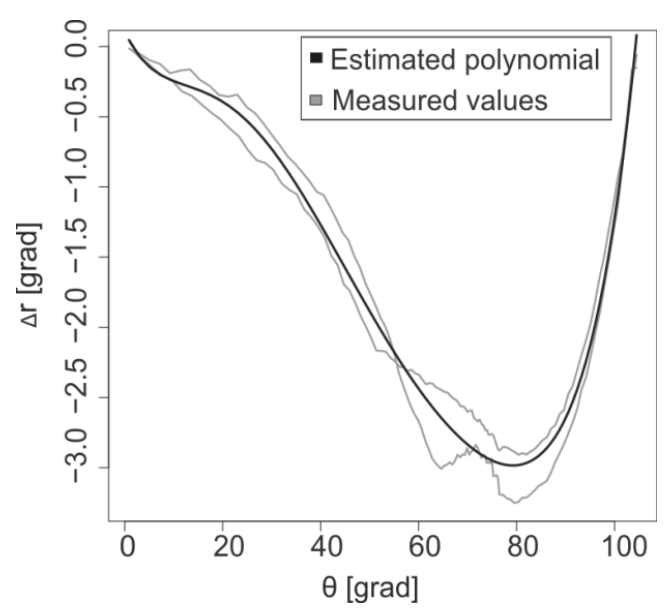

Fig. 9 Graph of the estimated radial distortion model polynomial.

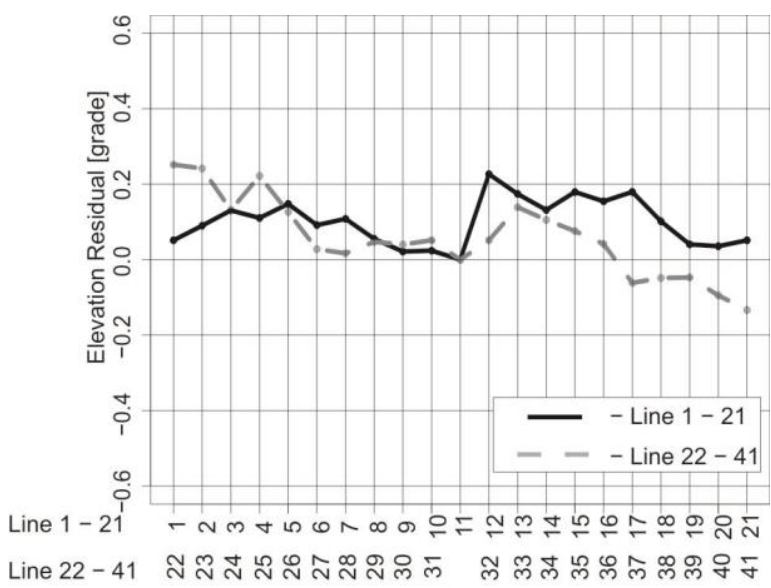

Point Number

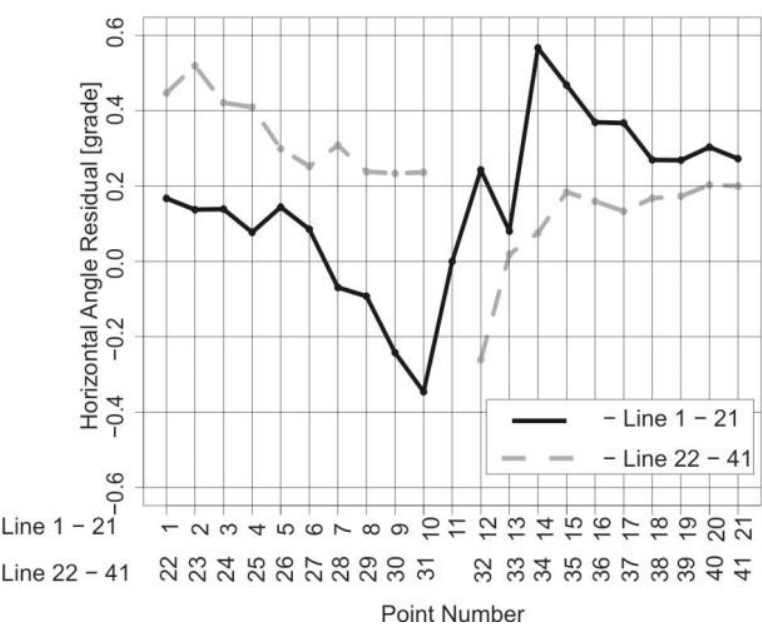

Fig. 10 Elevation and horizontal angle errors from laser scanning

images, reaching up to 4 grades (Fig. 11). The values of RMS for each image were almost two grades (Table 2).

The elevation angles reduction performed using previously determined polynomial allowed the error values to decrease to max. 2.2 grades. The values of 
Table 1 Summary of scanner measurements comparison.

\begin{tabular}{lccccc}
\hline & Min & Median & Mean & Max & RMS \\
\hline $\begin{array}{l}\text { Difference in } \\
\text { Hz angle }\end{array}$ & -0.3461 & 0.1999 & 0.1860 & 0.5667 & 0.1988 \\
$\begin{array}{l}\text { Difference in } \\
\text { elevation angle }\end{array}$ & -0.1336 & 0.0653 & 0.0782 & 0.2517 & 0.0882 \\
\hline
\end{tabular}

Table 2 RMS of elevation and horizontal angle.

\begin{tabular}{lccc}
\hline Image No & \multicolumn{2}{c}{ Error of elevation angle $[\mathrm{g}]$} & Error of horizontal angle [g] \\
\cline { 2 - 4 } & unreduced & reduced & unreduced \\
\hline 1 & 1.9274 & 1.1746 & 0.2857 \\
2 & 1.8724 & 0.8467 & 0.2523 \\
3 & 1.8440 & 0.7955 & 0.1394 \\
4 & 1.7810 & 0.8657 & 0.2317 \\
\hline
\end{tabular}

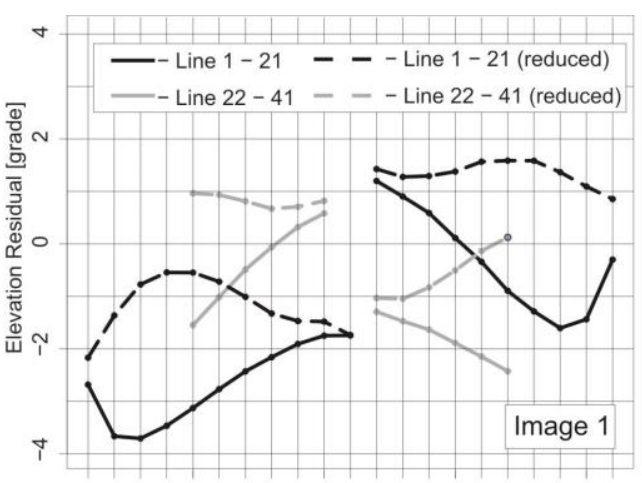

Line 1-21-Nmナเ -

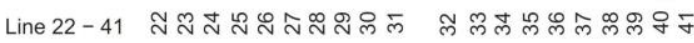
Point Number

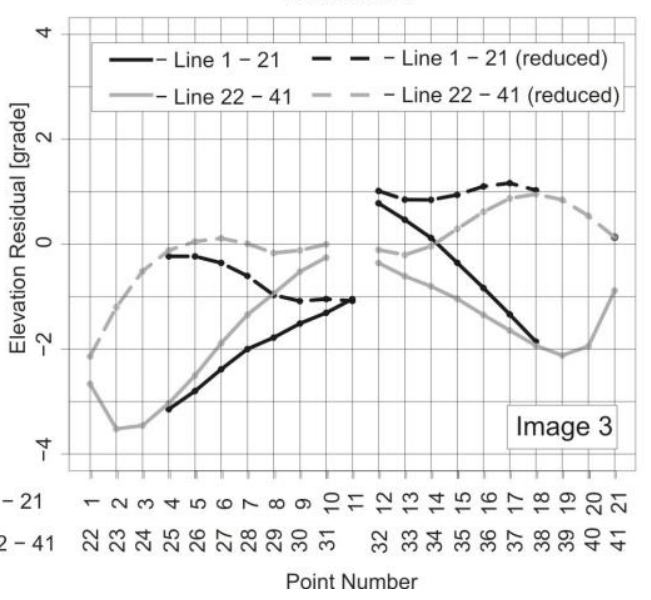

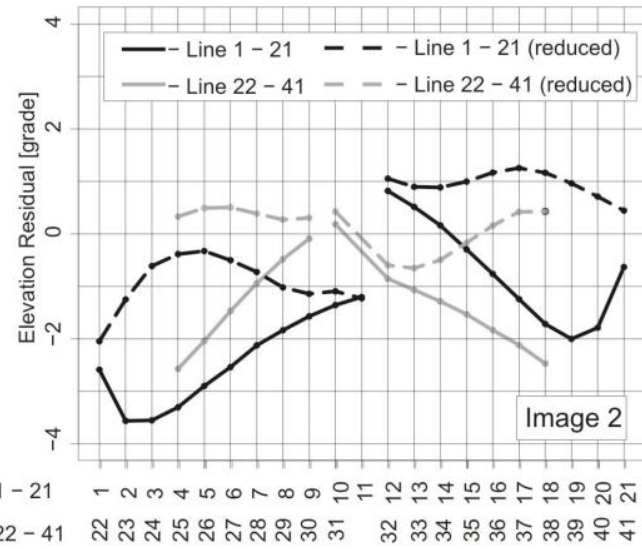

Point Number

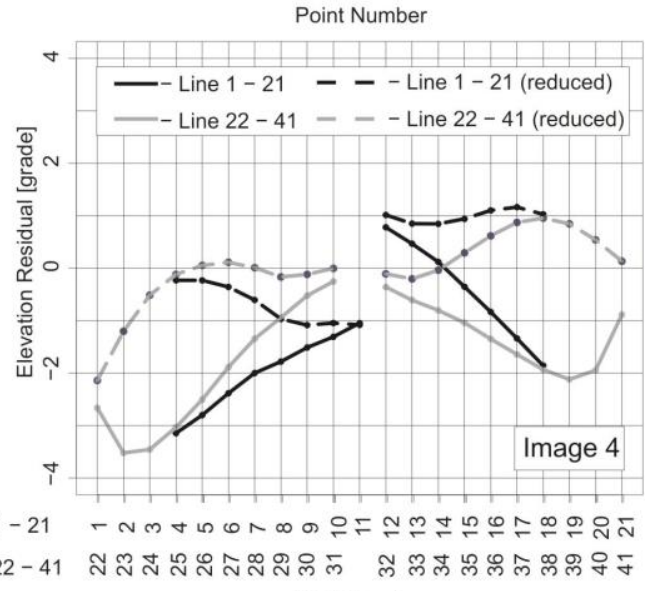

Point Number

Fig. 11 Elevation errors from hemispherical photography.

RMS for each image were in the range of 0.8 grades, except image 1, where RMS was 1.1746 grades.

Horizontal angle errors values, for unreduced readings, do not exceed 0.5 grade (Fig. 12) and values of RMS for each images slightly exceed 0.25 grade (Table 2). Just as in the case of elevation, errors of horizontal angles were increasing from the center toward the edges of the images. However, the values of horizontal angles errors are an order of magnitude lower than in case of elevation errors. Therefore the tangential distortions causing these errors were not adjusted in the presented research.

To sum up, using hemispherical photography as data collection method for GNSS mission planning, allow to obtain the accuracy of elevation and horizontal angle not worse than 2 and 0.5 grades respectively (Fig. 13). It should be noticed that measurements made on the edge of the image corresponding to the points at low elevations are affected the greatest errors. In practice, signals from 

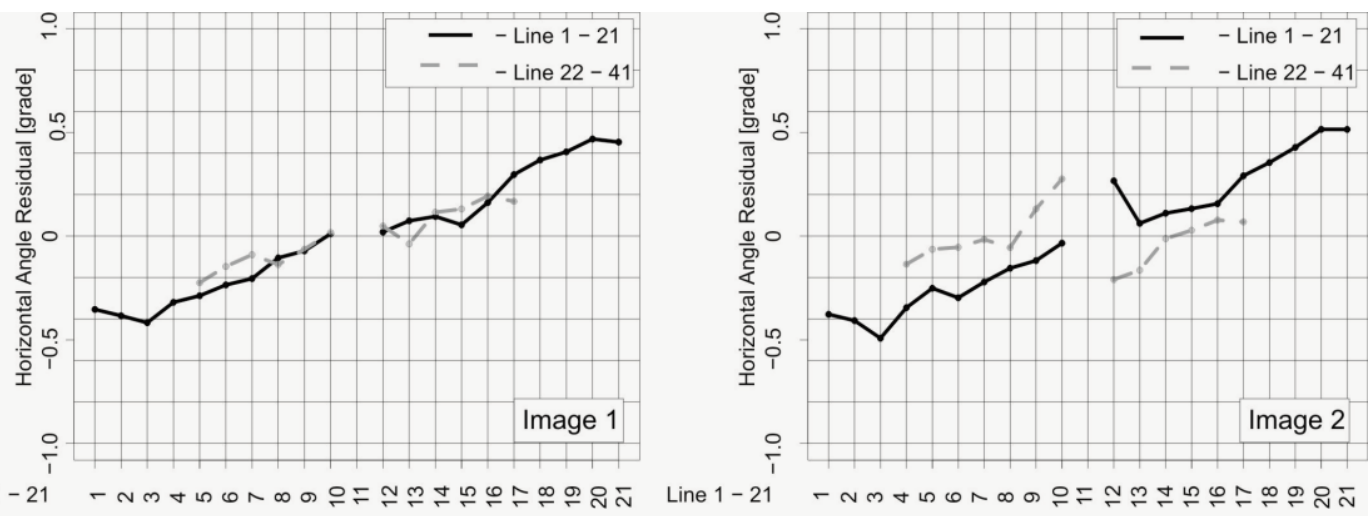

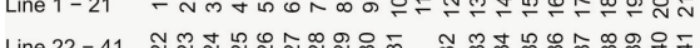

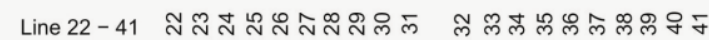

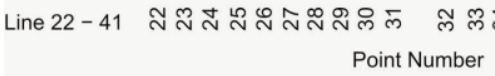
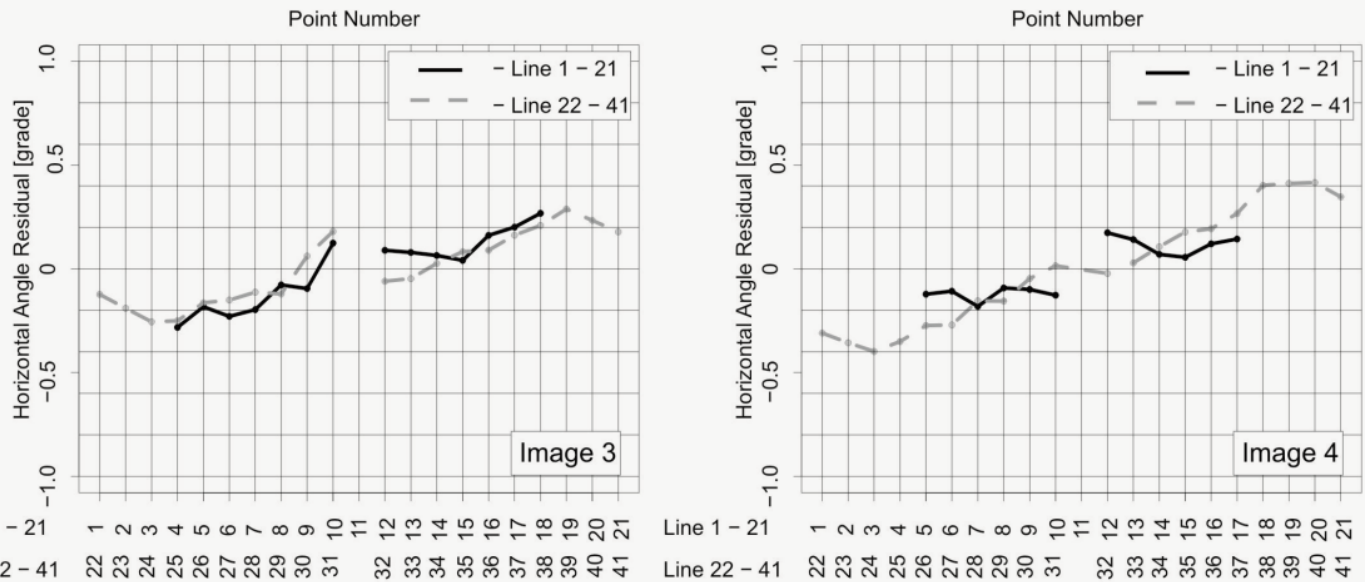

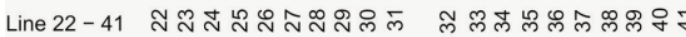

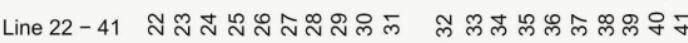

Fig. 12 Horizontal angle errors.

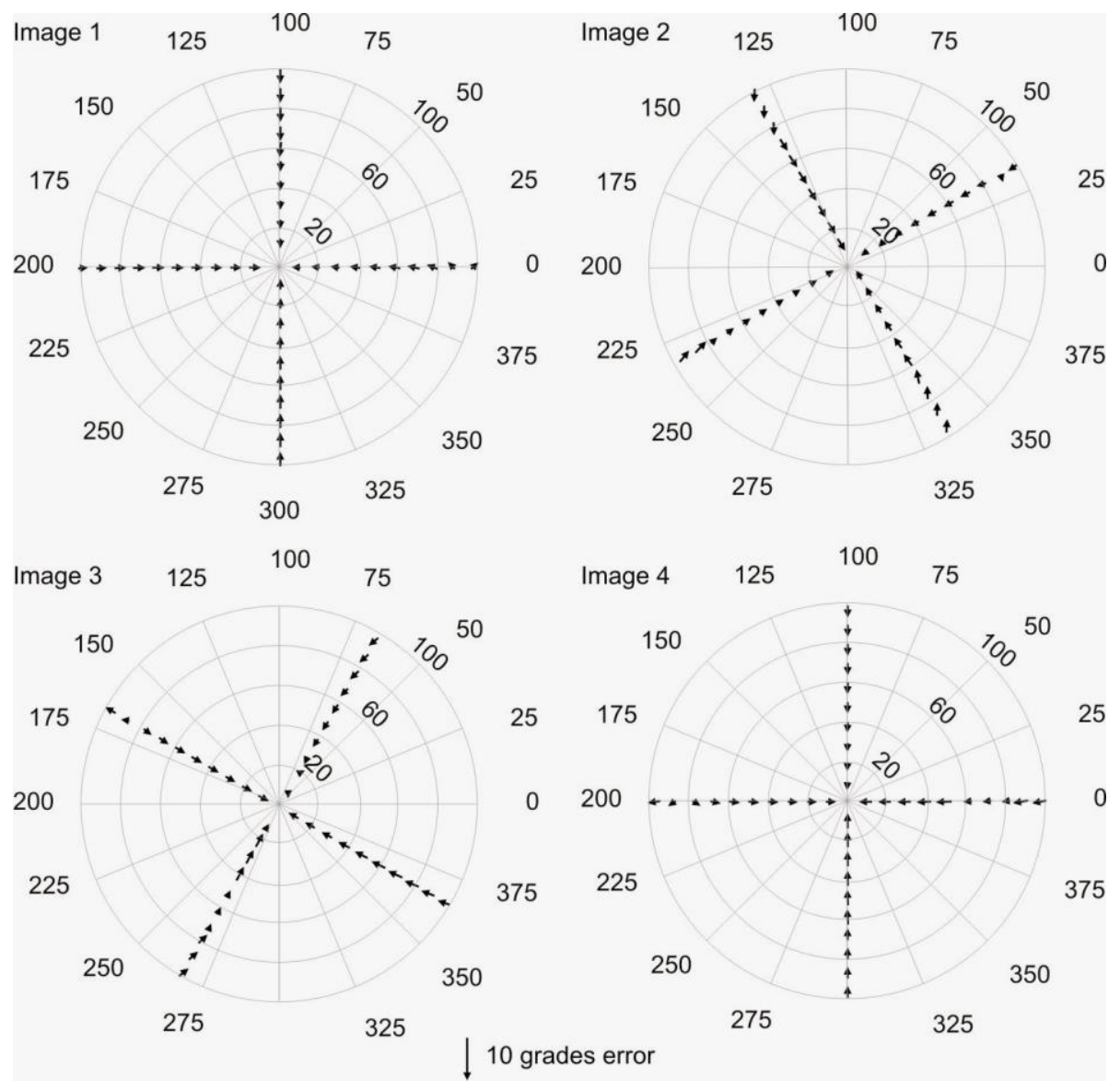

Fig. 13 Remaining residuals after reduction. 
low elevations satellites are not used to GNSS positioning that is why so called elevation mask is usually applied. Typically, that elevation mask is set in the range of 10 to 15 degrees. If the measurement results, which relate to control points located on the elevation less than 15 degrees will be removed from the measurement described above, obtained errors of elevation angles for the remaining points slightly exceed $1 \operatorname{grad}$ (except photo 1$)$.

\section{SUMMARY AND CONCLUSIONS}

In this paper two data acquisition methods were described and compared. Tests were performed using the hemispheric photography, and the laser scanner measurement. Tacheometric measurements were used as a reference.

The accuracy of the coordinates obtained from laser scanner was up to 0.5 grads for elevation angle and 0.2 grads for the horizontal angle, while errors of coordinates from hemispherical photography were two times higher and reached a value of 2 (point above 15 degree of elevation) and 0.5 grads respectively. Therefore it can be concluded that laser scanning technology is more suitable for terrain obstacles data acquisition. However, the cost of used equipment and the time that takes to process data should also be taken into account. The laser scanner is an expensive device and the development of results requires a lot of time, and special skills. Fieldwork with the scanner also takes some time. Taking a picture is much faster, photographic equipment costs are relatively lower and measurement elaboration is not difficult and can be partially automated. The only problem we encountered while taking a picture was leveling and centering, as neither the camera tripod nor the camera does have any suitable accurate equipment for this purpose.

\section{REFERENCES}

Ackermann, S., Angrisano, A., Del Pizzo, S., Gaglione, S., Gioia, C. and Troisi, S.: 2013, Digital surface models for GNSS mission planning in critical environments. Journal of Surveying Engineering, 140, No. 2, 040114001 DOI: 10.1061/(ASCE)SU.1943-5428.0000119

Bakuła, M., Pelc-Mieczkowska, R. and Walawski, M.: 2012, Reliable and redundant RTK positioning for applications in hard observational conditions. Artificial Satellites, Journal of Planetary Geodesy, 47, No. 1, 23-33.

Bakuła, M. and Pelc-Mieczkowska, R.: 2011, Reliable technique of GNSS/RTK positioning under severe observational conditions. Reports on Geodesy, 2(91), 67-73.

Beckschaefer, P., Seidel, D., Kleinn, C. and Xu, J.: 2013, On the exposure of hemispherical photographs in forests. iForest-Biogeosciences and Forestry, 6, 228237. DOI: 10.3832/ifor0957-006

Bitelli, G., Dubbini, M., and Zanutta, A.: 2004, Terrestrial laser scanning and digital photogrammetry techniques to monitor landslide bodies. International Archives of Photogrammetry, Remote Sensing and Spatial Information Sciences, 35(Part B 5), 246-251.

Boulianne, M., Nilette, C., Agnard, J.P. and Brindamour, M.: 1997, Hemispherical photographs used for mapping confined spaces.. Photogrammetric Engineering and Remote Sensing, 63, No. 9, 11031109.

Boulianne, M., Santerre, R., Gagnon, P. A. and Nolette, C.: 1996, Floating lines and cones for use as a GPS mission planning aid. Photogrammetric Engineering and Remote Sensing, 62, No. 3, 311-315.

Bulliner, E. and Hubbart, J. A.: 2013, An improved hemispherical photography model for stream surface shortwave radiation estimations in a central U.S. hardwood forest. Hydrological Processes, 27, No. 26, 3885- 3895. DOI: 10.1002/hyp.9524

Colcord, J. E.: 1989, Using fish-eye lens for GPS site reconnaissance. Journal of Surveying Enginiering, 115, No. 3, 347-352. DOI: 1061/(ASCE)0733-9453(1989)115:3(347)

D'Incà, D. and Zatelli, P.: 2004, New modules for satellite surveying planning in GRASS. Geomat Workb, 3, 114.

Federici, B., Giacomelli, D., Sguerso, D., Vitti, A. and Zatelli, P.: 2013, A web processing service for GNSS realistic planning. Applied Geomatics, 5, No. 1, 4557.

Gennery, D.B.: 2006, Generalizad camera calibration including fish-eye lenses. International Journal of Computer Vision, 68, No. 3, 239-266. DOI: $10.1007 / \mathrm{s} 11263-006-5168-1$

Gonsamo, A., D'Odorico, P. and Pellikka, P.: 2013, Measuring fractional forest canopy element cover and openness - definitions and methodologies revisited. Oikos, 122, No. 9, 1283-1291. DOI: 10.1111/j.1600-0706.2013.00369.x

Groves, P. D.: 2011. Shadow matching: A new GNSS Positioning Technique for urban canyons. Journal of Navigation, 64, No. 3, 417-430.

DOI: $10.1017 / \mathrm{S} 0373463311000087$

Grussenmeyer, P., Landes, T., Voegtle, T. and Ringle, K.: 2008, Comparison methods of terrestrial laser scanning, photogrammetry and tacheometry data for recording of cultural heritage buildings. International Archives of Photogrammetry, Remote Sensing and Spatial Information Sciences, 37(B5), 213-218.

Hugemann, W.: 2010, Correcting lens distortions in digital photographs, Ingenieurbüro Morawski+ Hugemann: Leverkusen, Germany.

Janowski, A., Nowak, A., Przyborski, M. and Szulwic, J.: 2014, Mobile indicators in GIS and GPS positioning accuracy in cities. Lecture Notes in Computer Science, LNAI8537, 309-318.

DOI 10.1007/978-3-319-08729-0_31

Kannala, J., and Brandt, S. S.: 2006, A generic camera model and calibration method for conventional, wideangle, and fish-eye lenses. Pattern analysis and machine intelligence, IEEE Transactions on, 28, No. 8, 1335-1340. DOI: 10.1109/TPAMI.2006.153

Krohn, D., Vonderhoe, A. and Mezera, D.: 1988, GPS on support of LIS: Project CONSOIL, Dane County, Wisconsin. Journal of Surveying Engineering, 114, No. 4, 172-181.

DOI: 10.1061/(ASCE)0733-9453(1988)114:4(172) 
Lichti, D. D. and Jamtsho, S.: 2006, Angular resolution of terrestrial laser scanners. The Photogrammetric Record, 21, No. 114, 141-160.

DOI: $10.1111 / j .1477-9730.2006 .00367 . x$

Luhmann, T., Robson, S., Kyle, S. and Harley, I.: 2006, Close-range Photogrammetry.

Parkinson, B. W. and Spilker, J. J.: 1996, Global Positioning System: Theory and Applications, Volume1. American Institute of Aeronautics and Astronautics, Inc.

Pelc-Mieczkowska, R.: 2014, Primary results of using hemispherical photography for advanced GPS mission planning. 9th International Conference Environmental Engineering (9TH ICEE) - Selected Papers.

DOI: 10.3846/enviro.2014.236.

Remondino, F. and Fraser, C.: 2006, Digital camera calibration methods: considerations and comparisons. IAPRS, XXXVI, Part 5, Dresden 25-27 September 2006.
Schwalbe, E.: 2005, Geometric modeling and calibration of fisheye lens camera systems. Proceedings 2nd Panoramic Photogrammetry Workshop, Berlin, Archives of Photogrammetry and Remote Sensing, ISPRS Archives, XXXVI, 5/W8.

Tardif, J. P., Sturm, P. and Roy, S.: 2006, Self-calibration of a general radially symmetric distortion model. $9^{\text {th }}$ European Conference on Computer Vision (ECCV), LNCS, 3954, 186-199. Springer Berlin Heidelberg.

Taylor, G., Li, J., Kidner, D., Brunsdon, C. and Ware, M.: 2007, Modelling and prediction of GPS availability with digital photogrammetry and LiDAR. International Journal of Geographical Information Science, 21, No. 1, 1-20.

DOI: $10.1080 / 13658810600816540$

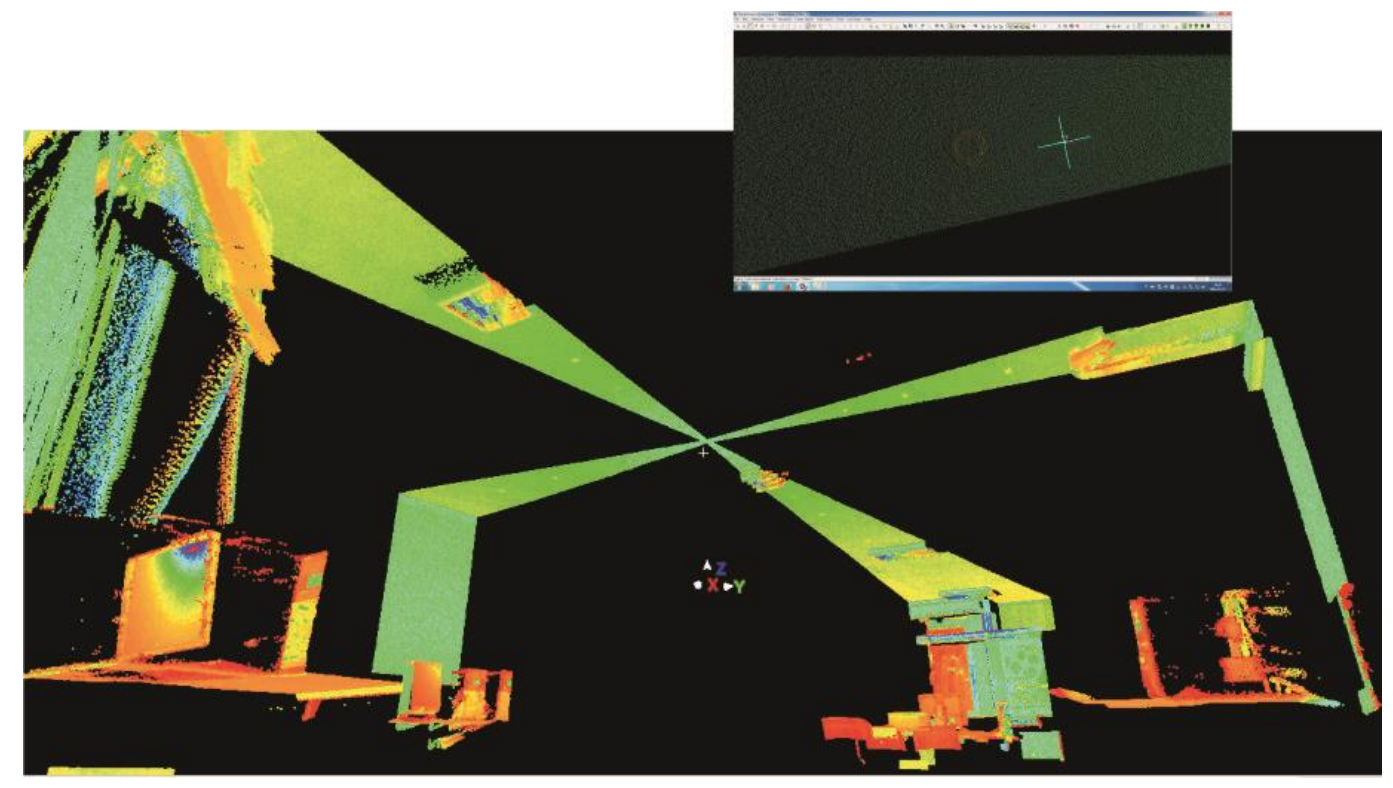

Fig. 5 Point cloud obtained from scanning of the test area. 